\title{
Effects of coupling to breakup channels in reactions induced by weakly bound and halo nuclei
}

\author{
J. P. Fernández-García ${ }^{1}$, A. Di Pietro ${ }^{1}$, P. Figuera $^{1}$, \\ M. Fisichella ${ }^{1}$, M. Lattuada ${ }^{1,2}$, A. M. Moro ${ }^{3}$, A. Musumarra ${ }^{1,2}$, \\ M. G. Pellegriti ${ }^{1,2}$, V. Scuderi ${ }^{1}$, D. Torresi ${ }^{1}$ and M. Zadro ${ }^{4}$ \\ ${ }^{1}$ INFN - Laboratori Nazionali del Sud, via S. Sofia 62, \\ 1-95123 Catania, Italy \\ 2 Dipartamento di Fisica e Astronomia, via S. Sofia 64, \\ I-95123 Catania, Italy \\ 3 Departamento de FAMN, Universidad de Sevilla, Apartado 1065, \\ E-41080 Seville, Spain \\ ${ }^{4}$ Ruder Bošković Institute, Bijenička cesta 54, HR-10000 Zagreb, Croatia
}

\begin{abstract}
The experimental elastic scattering angular distributions of the collisions involving the weakly bound nuclei ${ }^{6,7} \mathrm{Li}$ and the halo nucleus ${ }^{6} \mathrm{He}$ on the same ${ }^{64} \mathrm{Zn}$ target at several energies around the Coulomb barrier were measured at the Laboratori Nazionali del Sud (LNS, Italy), and the Cyclotron Research Center, Louvain La Neuve (Belgium), respectively. The experimental elastic scattering angular distributions of the ${ }^{6,7} \mathrm{Li}+{ }^{64} \mathrm{Zn}$ systems have been analyzed within the continuumdiscretized coupled-channels method, while the ${ }^{6} \mathrm{He}+{ }^{64} \mathrm{Zn}$ data have been compared with both continuum-discretized coupled-channels and coupled-reaction-channels calculations.
\end{abstract}

\section{Introduction}

In the last decade, the study of collisions involving halo and weakly bound nuclei had a notable interest. In fact, due to the coupling to breakup 
channels, strong effects on the elastic scattering and fusion have been observed $[1-4]$.

In the case of the stable lithium isotopes, ${ }^{6,7} \mathrm{Li}$, elastic scattering angular distributions on different targets have been extensively measured and analyzed within the Continuum-Discretized Coupled-Channel (CDCC) formalism, showing that the couplings to the continuum states have a significant effect on the elastic scattering involving the ${ }^{6} \mathrm{Li}$ nucleus [5].

One of the most studied exotic nuclei in the literature is ${ }^{6} \mathrm{He}$ (e.g. Refs. [6-11]), since such beam is available in different Radioactive Ions Beams (RIB) facilities with high intensity. The ${ }^{6} \mathrm{He}$ nucleus has a bound core $\left(\alpha\right.$ particle) and two loosely bound neutrons $\left(\mathrm{S}_{2 n}=0.97 \mathrm{MeV}\right)$. These two neutrons have a large probability of being far away from the core, creating the so-called nuclear halo. The ${ }^{6} \mathrm{He}$ nucleus constitutes an archetype of Borromean nucleus, since the subsystems $\alpha$-neutron and neutron-neutron are unbound.

Recently, a systematic study of ${ }^{6,7} \mathrm{Li}+{ }^{64} \mathrm{Zn}$ reactions at energies around the Coulomb barrier was performed at LNS. The elastic scattering angular distributions of both systems were measured at different energies $[12,13]$. We present results of the elastic scattering for the ${ }^{6,7} \mathrm{Li}+{ }^{64} \mathrm{Zn}$ reactions at the same center of mass energies. In addition, new preliminary experimental data for the ${ }^{6} \mathrm{He}+{ }^{64} \mathrm{Zn}$ collision are presented and compared with the threebody CDCC and Coupled-Reaction-Channels (CRC) calculations, assuming a simple di-neutron model of the projectile ${ }^{6} \mathrm{He}$.

\section{Experimental setups and results}

The ${ }^{6,7} \mathrm{Li}$ beams were generated by the SMP Tandem Van de Graaff accelerator and transported to the CT2000 scattering chamber at center of mass energies of 11.7, 12.4, 13.5, 15.0, 16.3 and $18.1 \mathrm{MeV}$. The experimental setup consisted in five silicon telescopes positioned on a rotating plate, in order to cover a wide angular range. The telescope systems were composed by a $10 \mu \mathrm{m}$ detector plus a $200 \mu \mathrm{m}$ detector. For the ${ }^{6} \mathrm{Li}+{ }^{64} \mathrm{Zn}$ reactions a ${ }^{64} \mathrm{Zn}$ target of $400 \mu \mathrm{g} / \mathrm{cm}^{2}$ was used [12], while a $70 \mu \mathrm{g} / \mathrm{cm}^{2}$ target for the ${ }^{7} \mathrm{Li}+{ }^{64} \mathrm{Zn}$ collisions.

On the other hand, the experiment corresponding to the ${ }^{6} \mathrm{He}+{ }^{64} \mathrm{Zn}$ reaction was carried out at the Cyclotron Research Center of Louvain La Neuve, Belgium. The reactions ${ }^{6,4} \mathrm{He}+{ }^{64} \mathrm{Zn}$ at center of mass energies of 13.5 and $16.5 \mathrm{MeV}$ were measured to continue old measurements performed by our group on the same systems at different energies $[3,14]$. The experimental 
setup was composed by an array of 4 LEDA detectors [15] covering the forward angles $5^{\circ}<\theta_{l a b}<12^{\circ}$. Another array of 3 LEDA detectors, placed at $45^{\circ}$ with respect to the beam axis, covered the angular range $22^{\circ}<$ $\theta_{l a b}<65^{\circ}$. Finally, two Double Sided Silicon Strips Detectors (DSSSD) were used to cover the backward angles, between $67^{\circ}$ and $120^{\circ}$. For additional details see Refs. [3,10].

The preliminary elastic scattering angular distributions of the ${ }^{6,7} \mathrm{Li}$ and ${ }^{6} \mathrm{He}$ on ${ }^{64} \mathrm{Zn}$ systems at the same center of mass energy $\left(\mathrm{E}_{c . m .}=13.5 \mathrm{MeV}\right)$ are presented in Fig. 1.

\section{Theoretical calculations}

In the last years, the CDCC method has been successfully applied to describe reactions induced by halo and weakly bound nuclei, such as ${ }^{6,7,11} \mathrm{Li},{ }^{11} \mathrm{Be}$ and ${ }^{6} \mathrm{He}[1,2,7,14,16-18]$. To solve the scattering problem, this formalism uses the coupled channel method. Due to the low breakup threshold, it is important to consider the continuum states of the weakly bound and halo nuclei. These states are included and discretized by means of the socalled binning method. The calculations were performed using the code FRESCO [19], in which both nuclear and Coulomb effects were considered.

The CDCC method is based on the elastic direct breakup mechanism, where the breakup process is considered as an inelastic excitation of the projectile to its continuum states. We would like to emphasize that this calculation do not consider other reaction mechanisms, such as neutron transfer or non-elastic breakup.

In this framework, we have analyzed the reactions induced by ${ }^{6,7} \mathrm{Li}$ and ${ }^{6} \mathrm{He}$ on the same ${ }^{64} \mathrm{Zn}$ target.

The ${ }^{6} \mathrm{Li}$ nucleus was described as an $\alpha+d$ structure, with a break-up threshold at $1.47 \mathrm{MeV}$ above the ground state. The resonances $3^{+}, 2^{+}, 1^{+}$ and the non-resonant states were included in the calculations. On the other hand, the ${ }^{7} \mathrm{Li}$ isotope was described as an $\alpha+t$ system, with a break-up threshold at $2.47 \mathrm{MeV}$. The excited state $1 / 2^{-}$, the resonances $7 / 2^{-}, 5 / 2^{-}$ and the non-resonant states were considered.

In Fig. 1, the solid red lines represent the CDCC calculations, while the dotted blue lines correspond to the calculations without couplings to the continuum states of the projectile. A good agreement between the predictions of the CDCC calculations and the experimental data was observed. In the case of the ${ }^{6} \mathrm{Li}$ induced collisions, it was necessary to include the couplings to the breakup channels to reproduce the experimental data, while 
for the ${ }^{7} \mathrm{Li}$ reactions these couplings were less important. This behavior could be due to the fact that the breakup threshold of ${ }^{6} \mathrm{Li}\left(\mathrm{S}_{\alpha}=1.47 \mathrm{MeV}\right)$ is smaller than that of ${ }^{7} \mathrm{Li}\left(\mathrm{S}_{\alpha}=2.47 \mathrm{MeV}\right)$.

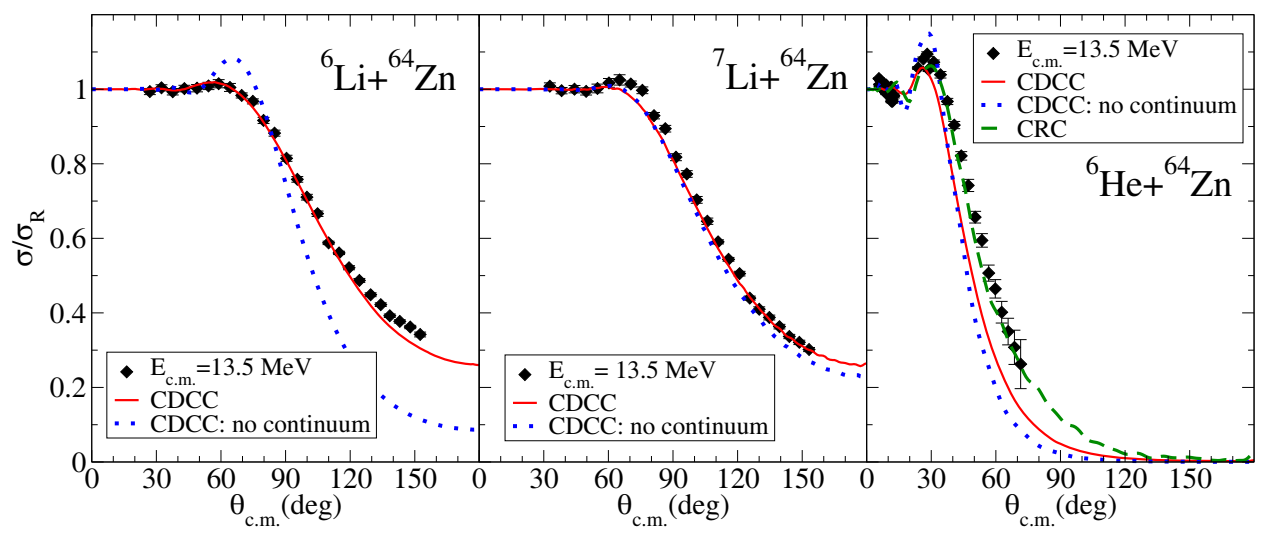

Figure 1: Preliminary elastic scattering angular distributions for the ${ }^{6,7} \mathrm{Li}$ and ${ }^{6} \mathrm{He}$ $+{ }^{64} \mathrm{Zn}$ collisions at the same center of mass energy $\left(\mathrm{E}_{c . m .}=13.5 \mathrm{MeV}\right)$. The CDCC calculations are represented by solid red lines, while the same calculation without the continuum couplings are shown by dotted blue lines. In the case of the ${ }^{6} \mathrm{He}+$ ${ }^{64} \mathrm{Zn}$ reaction, the $\mathrm{CRC}$ calculation is represented by the dashed green line.

The ${ }^{6} \mathrm{He}+{ }^{64} \mathrm{Zn}$ reaction has been analyzed within the three-body $\left(\alpha+2 n+{ }^{64} \mathrm{Zn}\right)$ CDCC method. The improved di-neutron model for ${ }^{6} \mathrm{He}$ $(\alpha+2 n)$ of Ref. [20] was considered. This model, which assumes a twoneutron cluster with spin zero bound to the $\alpha$ core with an effective separation energy of $1.6 \mathrm{MeV}$, was applied to describe several reactions involving ${ }^{6} \mathrm{He}$ nucleus [20].

In Fig. 1, the CDCC calculation of the elastic scattering angular distribution for the ${ }^{6} \mathrm{He}+{ }^{64} \mathrm{Zn}$ system is represented by the solid blue line. To study the effects of the coupling to the continuum states of the projectile, we have included the calculations without such couplings, represented by the dotted red line. Comparing both calculations we observe a strong effect of the couplings to breakup channels on the elastic cross section and a reduction of the Coulomb-nuclear interference peak.

However, the CDCC calculation underestimates the experimental data of the ${ }^{6} \mathrm{He}+{ }^{64} \mathrm{Zn}$ reaction. Therefore, additionally to this calculation, a Coupled Reaction Channel (CRC) calculation considering the one-neutron transfer to bound and unbound states of the target has been performed, since this calculation described the elastic cross section of the ${ }^{6} \mathrm{He}+{ }^{64} \mathrm{Zn}$ reaction at $\mathrm{E}_{c . m .}=12.4 \mathrm{MeV}[21]$. In this reference, the one-neutron transfer 
mechanism was found to be a more appropriate description than that considering two-neutron transfer to bound and unbound states of the target. In addition, the CRC calculations, performed in [21], were useful to describe the experimental fusion excitation function reported in Ref. [14].

The calculated elastic scattering angular distribution is represented by the green dashed line in Fig. 1. Unlike to the CDCC result, the CRC calculation improves the agreement with the experimental data.

The obtained result suggests that the elastic direct breakup process (CDCC calculation), where the core and the target are considered as inert particles, is not a good approach to describe the elastic scattering of the ${ }^{6} \mathrm{He}+{ }^{64} \mathrm{Zn}$ reaction at $\mathrm{E}_{c . m .}=13.5 \mathrm{MeV}$. On the other hand, the CRC calculation, based on a breakup process in which one neutron is transferred to bound and unbound states of the target, reproduces the experimental data. This result is different from that obtained in Ref. [21], where both CDCC and CRC calculations were in reasonable agreement with the experimental data at $\mathrm{E}_{\text {c.m. }}=12.4 \mathrm{MeV}$, which suggests a possible energy dependence of the different breakup mechanisms.

\section{Conclusions}

Experimental results for elastic scattering of the weakly bound nuclei ${ }^{6,7} \mathrm{Li}$ and the halo nucleus ${ }^{6} \mathrm{He}$ on ${ }^{64} \mathrm{Zn}$ at the same center of mass energy have been presented.

CDCC calculations for the ${ }^{6,7} \mathrm{Li}+{ }^{64} \mathrm{Zn}$ collisions have been performed, showing a good agreement with the experimental data. The couplings to the breakup channels have an important effect in the case of the ${ }^{6} \mathrm{Li}$ scattering, whereas minor effects have been found for the ${ }^{7} \mathrm{Li}$ case. This behavior could be linked to the smaller binding energy of the ${ }^{6} \mathrm{Li}\left(\mathrm{S}_{\alpha}=1.47 \mathrm{MeV}\right)$ nucleus compared with ${ }^{7} \mathrm{Li}\left(\mathrm{S}_{\alpha}=2.47 \mathrm{MeV}\right)$.

In the ${ }^{6} \mathrm{He}+{ }^{64} \mathrm{Zn}$ reaction, we have observed a strong effect of the coupling to breakup channels. Two different calculations, CDCC and CRC calculations, including couplings to the breakup channels, have been performed within different breakup mechanism. The CDCC calculation fails to describe the experimental elastic scattering angular distribution. However, we have observed that the CRC method provides a more suitable approach to describe the elastic scattering angular distribution, where the ${ }^{6} \mathrm{He}$ breakup is treated within one-neutron transfer to the continuum picture. 


\section{References}

[1] Di Pietro A. et al. Phys. Rev. Lett., 105 (2010) 022701.

[2] Cubero M. et al. Phys. Rev. Lett., 109 (2012) 262701.

[3] Scuderi V. et al. Phys. Rev. C, 84 (2011) 064604.

[4] Canto L. F. et al. Nucl. Phys. A, 821 (2009) 51.

[5] Canto L. F., Gomes P. R. S., Donangelo R., and Hussein M. S. Phys. Rep. 424 (2006) 1.

[6] Sánchez-Benítez A. M. et al. Nucl. Phys. A, 803 (2008) 30.

[7] Acosta L. et al. Phys. Rev. C, 84 (2011) 044604.

[8] Aguilera E. F. et al. Phys. Rev. C, 63 (2001) 061603.

[9] Kakuee O. R. et al. Nucl. Phys. A, 728 (2003) 339.

[10] Di Pietro A. et al. Europhys. Lett., 64 (2003) 309.

[11] Fernández-García J. P., Rodríguez-Gallardo M. , Alvarez M. A. G., and Moro A. M. Nucl. Phys. A, 840 (2010) 19.

[12] Zadro M. et al. Phys. Rev. C, 80 (2009) 064610.

[13] Zadro M. et al. Phys. Rev. C, 87 (2013) 054606.

[14] Di Pietro A. et al. Phys. Rev. C, 69 (2004) 044613.

[15] Davinson T. et al. Nucl. Inst. and Meth. A, 454 (2000) 350.

[16] Fernández-García J. P. et al. Phys. Rev. Lett., 110 (2013) 142701.

[17] Sakuragi Y. Phys. Rev. C, 35 (1987) 2161.

[18] Beck C., Keeley N., and Diaz-Torres A. Phys. Rev. C, 75 (2007) 054605.

[19] Thompson I. J. Comp. Phys. Rep. 7 (1988) 167.

[20] Moro A. M., Rusek K., Arias J. M., Gómez-Camacho J. G. and Rodríguez-Gallardo M. Phys. Rev. C, 75:064607, 2007.

[21] Moro A. M., Fernández-García J. P., Alvarez M. A. G., and RodríguezGallardo M. EPJ Web of Conferences, 17 (2011) 08001. 